\title{
Einführung Teil 2: Statistiken 2005
}

\section{Gérard Perroulaz and Xavier Tschumi Canosa}

\section{OpenEdition}

\section{Journals}

Electronic version

URL: http://journals.openedition.org/sjep/199

DOI: 10.4000/sjep.199

ISSN: 1663-9677

\section{Publisher}

Institut de hautes études internationales et du développement

\section{Printed version}

Date of publication: 1 avril 2005

Number of pages: 215

ISBN: 2-88247-058-4

ISSN: $1660-5926$

\section{Electronic reference}

Gérard Perroulaz und Xavier Tschumi Canosa, «Einführung Teil 2: Statistiken 2005 », Schweizerisches Jahrbuch für Entwicklungspolitik [Online], 24-1 | 2005, Online erschienen am: 22 April 2010, abgerufen am 25 September 2020. URL : http://journals.openedition.org/sjep/199 ; DOI : https://doi.org/ 10.4000/sjep.199 


\section{Einführung}

M ZWEITEN TEIL des Schweizerischen Jahrbuchs für Entwicklungspolitik (Nr. 1) werden üblicherweise die wichtigsten Zahlenangaben zu den Beziehungen der Schweiz mit den Entwicklungs- und Transitionsländern vorgestellt. Dabei werden drei Hauptkategorien dieser Beziehungen - Handelsströme, Finanzflüsse und Entwicklungshilfeströme - untersucht.

In Anbetracht der Internetverbreitung und der zunehmenden Verfügbarkeit statistischer Daten, die jedem Internetbenutzer zugänglich sind, wird dieser zweite Teil des Jahrbuchs ab diesem Jahr in einer neuen Form präsentiert :

๖ Der erste Abschnitt (Teil A) ist als Anleitung für die Leserinnen und Leser gedacht, um im Internet die wichtigsten Informationen zu den drei bislang im Statistischen Teil des Jahrbuchs behandelten Kategorien von Flüssen aufzufinden.

ـ Der zweite Abschnitt (Teil B) vermittelt einen Überblick über die Beziehungen der Schweiz zu den Entwicklungs- und Transitionsländern und übernimmt die Tabellen des 4. Teils der Statistik der vorhergehenden Jahrbuchausgaben.

๖ Der dritte Abschnitt (Teil C) stellt gewisse für diese Beziehungen charakteristische Ströme heraus. Je nach Wahl der betreffenden Ströme wird die Präsentation dieses Teils von Jahr zu Jahr verschieden sein.

Die neue Aufmachung des Statistischen Teils stellt unbestreitbar einen Bruch gegenüber der bisherigen Form dar. Einen solchen Bruch zu beschliessen, war nicht selbstverständlich. Wenn dieser Beschluss getroffen wurde, so hauptsächlich aufgrund der Feststellung, dass eine Anzahl Tabellen, die bislang im Statistischen Teil des Jahrbuchs aufgeführt wurden, sich derzeit in gleicher oder sehr ähnlicher Form im Internet befinden. Deshalb werden von jetzt an lediglich Tabellen mit Daten, die gegenüber den Internetdaten einen Mehrwert bieten, im Statistischen Teil des Jahrbuchs veröffentlicht.

Folglich wäre das Redaktionskomitee des Jahrbuchs den Benutzerinnen und Benutzern des Statistischen Teils dankbar, ihre Bemerkungen zu dem hier präsentierten neuen Aufbau, sofern sie dies wünschen, an <xavier.tschumi@ iued.unige.ch $>$ oder < gerard.perroulaz@iued.unige.ch $>$ zu richten. 